\title{
SPIRAL: A novel biologically-inspired algorithm for gas/odor source localization in an indoor environment with no strong airflow
}

\author{
Gabriele Ferri ${ }^{\mathrm{a}, \mathrm{b}, *}$, Emanuele Caselli ${ }^{\mathrm{a}, \mathrm{b}}$, Virgilio Mattoli ${ }^{\mathrm{b}}$, Alessio Mondini ${ }^{\mathrm{b}}$, Barbara Mazzolai ${ }^{\mathrm{b}}$, \\ Paolo Dario ${ }^{\mathrm{b}}$ \\ a BioRobotic Engineering School, IMT Lucca Institute for Advanced Studies, Piazza San Ponziano 6, 55110 Lucca, Italy \\ b CRIM Laboratory, Scuola Superiore Sant'Anna, Viale Rinaldo Piaggio 34, 56025 Pontedera (Pisa), Italy
}

\section{A R T I C L E I N F O}

Article history:

Received 26 July 2007

Received in revised form

3 July 2008

Accepted 15 July 2008

Available online 20 July 2008

\section{Keywords:}

Indoor monitoring

Gas source localization

Bio-inspired algorithm

\begin{abstract}
A B S T R A C T
This work describes the design and experimental results of an algorithm, designed to localize a gas source in an indoor environment with no strong airflow by using an autonomous agent. This condition exacerbates the patchiness and intermittency of odor distribution, typical of turbulent flows in the presence of strong mean flows. Furthermore, no information about the wind can be used to detect the position of the source. In the approach proposed here, the robot moves along spirals. A spiral can be reset and a new one started, based on the information acquired about gas distribution. This enables the robot to get close to the ejecting source, without relying on airflow measurements. Results from experiments are also described and discussed, to assess the efficiency of the proposed method.
\end{abstract}

(c) 2008 Elsevier B.V. All rights reserved.

\section{Introduction}

This paper describes the design and implementation on a robotic autonomous agent of a biologically-inspired algorithm, named SPIRAL (Searching Pollutant Iterative Rounding ALgorithm), which aims to localize a gas/odor source in an indoor environment in the absence of a strong and constant airflow. Even if this condition represents a realistic indoor scenario very well, it presents certain difficulties to the autonomous agent to track a plume up to its source. The lack of a strong wind exacerbates the patchiness and intermittency of gas distribution, as it is typical of turbulent flows in the presence of strong mean flows. Gas dispersion is characterized by low energy, turbulent mixing and weak convective airstreams. Therefore, gas distribution presents high temporal variations around an average value. Gas distribution features and the lack of possibility of using information about wind direction to infer the position of an emitting source, make these experimental conditions much more challenging than the experimental setting usually used in literature for plume tracking experiments, where a strong and constant wind is artificially generated.

In the approach here pursued, the robot moves along a spiral path and stops at a temporal window in some specific locations

\footnotetext{
* Corresponding author at: CRIM Laboratory, Scuola Superiore Sant'Anna, Viale Rinaldo Piaggio 34, 56025 Pontedera (Pisa), Italy. Tel.: +39 050883417; fax: +39 050883497.

E-mail address: g.ferri@imtlucca.it (G. Ferri).
}

in order to sample the gas. Based on the features collected from the information acquired, the robot computes an index, called the Proximity Index (PI), to assess how close the source of the gas is to the sampling location. The PI is computed based on the average of the signal measured, together with a measurement of the peaks present in the acquired signal (concentration peak frequency and intensity seem to increase in association with proximity to a source $[3,22])$. Based on considerations about the created PIs, a spiral may be reset and, after that, a new one is started. This movement allows the robot to approach an emitting source without relying on any information about the airflow.

The SPIRAL algorithm was implemented on a robotic platform developed by the authors $[9,10]$ and experimental results are reported here. To test the efficiency of the algorithm we also evaluated the results of a so-called random SPIRAL, in which the original SPIRAL algorithm was tested with randomly created PIs. We have also compared SPIRAL with a bacterium inspired algorithm [32], with two different sampling times, to investigate how changes in the acquisition time may affect its behavior.

The paper is outlined as follows: the next section focuses on the potentialities and difficulties associated with the localization of a chemical source by using an autonomous robot. A review follows of previous works on source localization in experimental settings with and without a strong wind. Section 2 illustrates sensors calibration and source characterization results. Section 3 introduces the SPIRAL algorithm. Section 4 and 5, respectively, describe the experimental set-up and experimental results. Finally, conclusions and recommendations for future research close the paper. 


\subsection{Searching for a gas/odor source by using an autonomous robotic agent}

The sense of smell is widely used throughout the biological world. Animals use odors to catch information about the environment and to appropriately react to different situations [7]. Odor is fundamental for many animal species to localize a possible source of food $[5,13,34]$ a mate (like moths, which follow a pheromone plume $[2,6])$, or to escape from possible predators [15]. Furthermore, odors can also be used as a means of communication to organize complex social behaviors: bees use odor to recognize relatives or nest-mates [14], and ants use trails of pheromone to coordinate movements and improve the ability for the swarm to find food [8], just to give a few examples.

The ability to smell volatile chemicals provided by these sensors has lead researchers - from the 90s on - to investigate the use of gas sensors for robot navigation. The ability of robots to orientate themselves by smelling some odor has proved useful in many scenarios. Two applications have been investigated so far: a robot using a trail of odor marked on the ground, to follow a particular path [30], or a robot using odor clues to localize an emitting source. In this paper, we will focus on the second issue. Gas source localization by using an autonomous agent would help us to accomplish many tasks, in either indoor or outdoor environments: it could help to localize leaks of harmful gas in industrial or domestic environments, to monitor a poisoned area, to detect pipeline leakages and to search for hidden explosives or narcotics.

To localize an emitting chemical source in a rapid and reliable way is not an easy task, because of the complex nature of gas distribution. Gas source localization essentially involves three different subtasks, and according to the taxonomy of [16], they include the following: plume finding - namely detecting increased gas concentration, plume traversal - namely following the plume up to the source, and source declaration - namely determining if a source has been found. Turbulent gas dispersion brings up a few issues for autonomous agents when fulfilling the above mentioned subtasks. At medium and high Reynolds numbers (the situation encountered by an autonomous agent), gas dispersion is dominated by turbulent mixing [4,33]. Odor is concentrated in "packets", often with extremely low concentration measured between immersions in an odor packet. The slow velocity of gas molecules to diffuse (ethanol constant diffusion is $0.119 \mathrm{~cm}^{2} / \mathrm{s}$ at $25{ }^{\circ} \mathrm{C}$ and $1 \mathrm{~atm}$ with a diffusion length in one hour calculated to be only $20.7 \mathrm{~cm}$ ) implies that gas dispersion is totally dominated by airflows: flow turbulence dominates the distribution of gas molecules. As a consequence, instantaneous gradients are poorly defined, time varying and frequently do not point towards the source [28]. To solve these problems, most researchers test their approaches by introducing an artificial strong airflow in the experiment area, to minimize the effects of gas turbulent transport. Therefore, tracking a gas plume up to its source with no sufficiently strong airflow is a much more difficult task: gas dispersion is dominated by low energy turbulent mixing and weak convective flows, produced by spatial temperature differences [35], resulting in a patchier and more fluctuant gas distribution. Local concentration maxima and even the absolute maximum have been observed at some distance from the source, if the source was active for some time [24].

The two different conditions (absence and presence of a strong and constant wind) are shown in Figs. 2 and 3, which report our source characterization test results, namely of one source ejecting ethanol in the presence and absence of a strong wind (see Section 2).

Test results show the following:

- With a strong and constant wind, at least in an area close to the source, low fluctuations around the average gas value are noticed and a gradient is defined (Fig. 2);
- Without any wind, high fluctuations of gas concentration occur around the average value (Fig. 3).

Therefore, introducing a constant and strong airflow provides several benefits in localizing a chemical source. Gas concentration is temporally less fluctuant; the plume consists of two areas, as noticed in [12,20]: a region closer to the source and a far region. The region closer to the source consists of a high gas concentration odorant, which sharply falls off at the edges of the plume (and which makes the plume finding task easier for a robot), with the average concentration decreasing when going downwind: here a concentration gradient can be detected. Going downstream, the plume becomes larger and edges are less well defined. This is the far region, where instantaneous concentration is highly variable. Moreover, the direction and intensity of a strong wind can be easily measured by using an anemometer, where wind direction may help to track the plume up to its source.

In the next section, we will review the approaches proposed by researchers in the two different scenarios.

\subsection{Previous works in an indoor environment in the presence and in the absence of a strong and constant airflow}

In experimental conditions with a strong and constant airflow, gradient-ascent based methods have been proposed and tested [20,26,32]. In these works the robot uses spatially-separated gas sensors. It reacts to a sensed concentration gradient moving towards the direction in which it senses a higher gas intensity. Based on this strategy, a wheeled underwater robot was also made to move in a flume featuring an artificially generated flow [12]. Results show that the gradient can be followed if the robot moves inside the artificially generated plume and it is not too far from the source: here a gradient is detectable. If the robot gets outside the well defined plume or far from the source, the information acquired is misleading and the robotic agent is liable to get "stuck" into local maxima or to wander about.

The biological world has inspired a family of bio-inspired algorithms aimed at reproducing the successful behaviors of animals in localizing ejecting sources. The chemotactic behavior of the bacterium Escherichia coli has been implemented and tested on robots $[26,27,32]$. The bacterium strategy works well in a small scale, where the chemical dispersion is characterized by diffusion. At large scales it presents the same flaws as the gradient-ascent based algorithms: the robot is liable to wander about if it is far from the source or outside the well defined edges of the plume. However, unlike the gradient-ascent techniques, it is not affected by the difficulties of relying on differential measurements taken with different sensors, which are often misleading because of the intermittent nature of the plume. The most studied behavior is no doubt the upwind flight of the male moth searching for a female ejecting pheromone [2,6]. The behavior of the moth consists of counterturning patterns with alternate turns to the left and right, according to the wind direction (casting), to come into contact with packets of odor, and then an upwind movement (surge), to come closer to the source. This successful behavior was theoretically studied [4] and implemented on robots [27,29, 32 ], showing its efficiency and reliability. Other approaches aim at using information about wind direction and gas samplings, to mix plume acquisition and plume following behaviors up to a source $[16,17,31]$.

Much less work has addressed indoor environments without artificially created strong ventilation, despite the fact that this condition is more realistic and common in indoor environments, and that this is the actual situation in which robots will have to work in real applications. 
As previously anticipated, without a strong and constant airflow, gas concentration presents peaks and high variability, with respect to the average value (see Fig. 3). The extremely weak airflows present in a room are hardly detectable. In fact, the classically used thermal airflow anemometers show detection limits ( $5 \mathrm{~cm} / \mathrm{s}$ ), unable to detect weak airflows [19]. Also the more sensible ultrasonic anemometers (detection limits of about $1 \mathrm{~cm} / \mathrm{s}$ ) sometimes fail to detect wind in particular areas of a room [19]. In addition, the movement of the robot can have some significant impact in measuring weak airflow. Finally, it is worth noting that, also with a constant mean airflow, the presence of obstacles can generate areas where information about the wind cannot be used by the robot. To deal with this issue, some researchers have tried to propose methods that either do not rely on or only partially rely on wind measurements.

Experiments with a robot moving in a corridor are reported in [21], to investigate the relation between gas measurements and source location (peaks in measured concentration are observed to be associated with a source nearby). In [18] the use of gas sensors, together with a vision system to identify some possible ejecting objects, is tested in a room without forced ventilation: the information about the airflow is only used when it is available. However, the vision can be useful only if the source is a recognizable object, for example a bin or a bottle. If it is a leak from a pipeline, the vision cannot help to localize the source. In [23], a modified moth behavior is described which does not use wind information for its peculiar upwind movement.

With no wind, gradient-ascent based techniques can improve overall performances, but they may be easily affected by extremely multimodal gas distribution [22].

\section{Sensor calibration and source characterization}

As odor sensors, we chose TGS 800 commercial sensors manufactured by Figaro Engineering Inc. They are metal oxide semiconductor gas sensors, and, in the presence of alcohol vapors, internal resistance changes, based on a logarithmic function. This kind of sensor was chosen for its high sensitivity, usable long life span, and comparatively high robustness to changing environment conditions [25]. The sensors are small, cheap, and can be easily integrated in the measurement circuit [25]. Their main drawbacks involve the need for a high operating temperature, which causes a high power consumption and slow recovery time after the gas is removed. Furthermore, they include a variance of the response characteristics between individual sensors. For this reason, at first, some calibration sessions were carried out. Sensors (6 sensors: 5 for source characterization, 1 used on the robot) were tested in a hermetically closed box, where a known increasing amount of alcohol was periodically injected. A fan was also included to accelerate alcohol vaporization and homogeneous dispersion. The signals coming from the sensors were acquired using a DAQCard$6024 \mathrm{E}^{\mathrm{TM}}$, from National Instruments. Data were sampled every $1 \mathrm{~ms}$, and each stored value was the mean calculated on 500 measurements. In order to calibrate the sensors, the data collected were fitted with the characteristic bi-logarithmic function given by the sensors datasheet: the sensed gas concentration is proportional to the value of the internal resistance, that is,

$$
\log (c)=f\left(\log \left(R / R_{0}\right)\right)
$$

where $c$ is the sensed gas concentration, $R$ is the internal resistance and $R_{0}$ is the resistance when no gas is sensed by the sensor.

We fitted this relation for all the known concentrations injected in the box $\left(c_{i}\right)$ (we used 14 concentrations) using a third degree polynomial:

$$
\log \left(c_{i}\right)=k_{0}+k_{1} \log \left(R / R_{0}\right)+k_{2} \log \left(R / R_{0}\right)^{2}+k_{3} \log \left(R / R_{0}\right)^{3}
$$

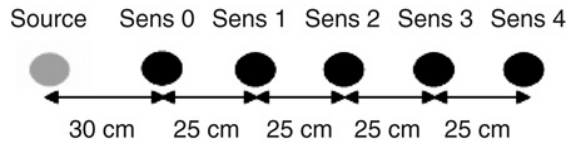

Fig. 1. Position of the sensors related to the ethanol source during the experiments for source characterization. The source was a circular $8.5 \mathrm{~cm}$ diameter dish containing ethanol alcohol.

where $c_{i}$ are the known ethanol concentrations used during the calibration session and $k_{i}$ are the coefficients of the fitting polynomial. We solved, in the least squares sense, the systems of equations for the different sensors, to find the desired polynomial coefficients. The coefficients of the polynomial, characteristic of each sensor, were used to convert sensor voltage outputs into concentrations.

In order to characterize gas dispersion, some preliminary experiments were carried out. The source used was an $8.5 \mathrm{~cm}$ diameter circular dish, containing ethanol alcohol, because of its volatility at room temperature and no toxicity. This is the same source used in the experiments with the robot. We used the previously calibrated sensors to measure alcohol concentrations. We used 5 sensors placed in a row, spaced $25 \mathrm{~cm}$ from each other. The first one is at a distance of $30 \mathrm{~cm}$ from the source (see Fig. 1). The acquisitions started after the ethanol had been free to evaporate for $15 \mathrm{~min}$. Two different kinds of trials were carried out: one without the introduction of an external artificial airflow and a second one with a fan, placed $40 \mathrm{~cm}$ behind the source, generating an airflow of about $50 \mathrm{~cm} / \mathrm{s}$. We collected data placing the sensors in four perpendicular directions to investigate the spatial differences in gas distribution. Figs. 2 and 3 report the results for one direction. Fig. 2 reports the results with the sensors placed inside the limits of the well defined generated plume.

The figures clearly show the differences between the two conditions. With the presence of the wind, at least inside the well defined plume and not too far from the source, gas concentration is much smoother: a gradient is detectable, low variations around an average value are present. There is a sharp fall in concentration at the edges of the plume, created by the forced ventilation. The analysis of Fig. 3, however, clearly shows a much more complex distribution. There are high variations around an average value, and at a distance from the source it appears more difficult to recognize which sensor is the one closest to the source. The gas distribution shows much more spatial uniformity than for the case with wind, in which extremely low concentrations are present on the upwind side of the source. Fig. 3 also shows an interesting feature of gas distribution: getting closer to the source, peaks of gas intensity become more frequent and higher. These peaks, also noted in $[3,22]$, appear to be a distinctive feature of the proximity to an emitting source. It has also been suggested that some animals (lobsters, for example) [3] might theoretically be able to extract an odor landscape from the shapes of the peaks of sensed odor, and use it to move up to a food source.

\section{SPIRAL algorithm}

SPIRAL is an algorithm to localize a chemical source without using any information about the airflow, so as to be able to work reliably in an indoor environment with no strong airflow. The SPIRAL idea involves spiral movements. During the spiral movements the robot stops to sample gas. With the data acquired, it figures out its proximity to the source: if it finds out it is closer to the source in comparison with the previous measurements, the robot starts one more spiral movement, otherwise, it continues in the current one. This way, spirals are propagated to get closer to the source (see Fig. 4). Fig. 5 shows the actual spiral movement that the 


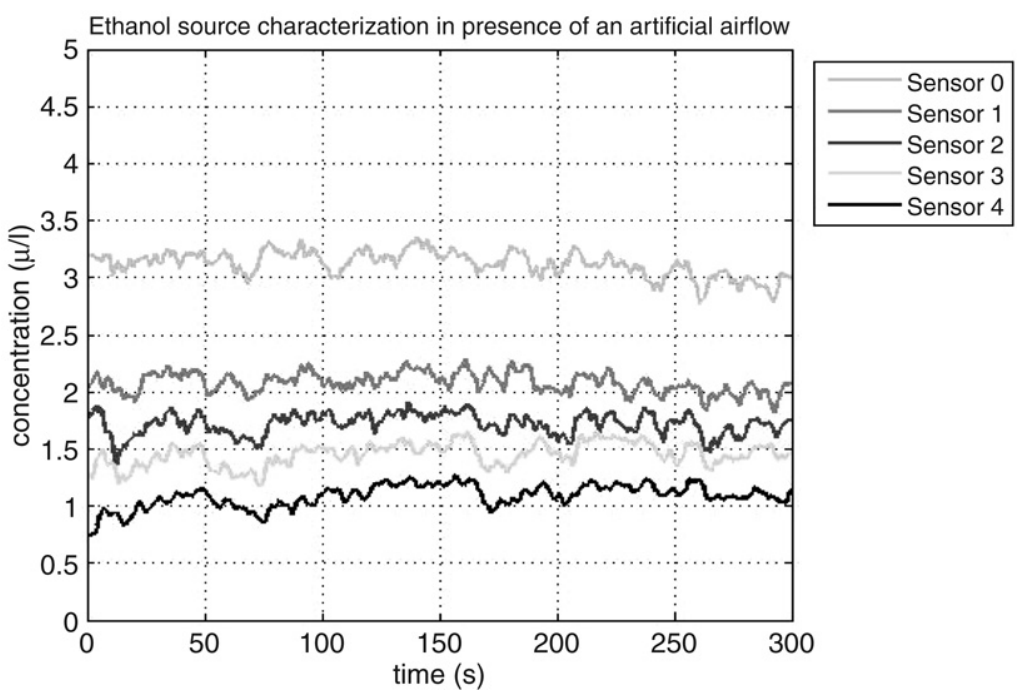

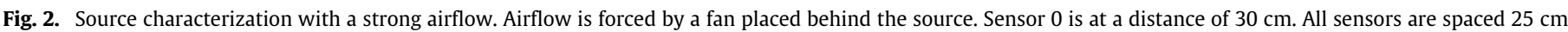

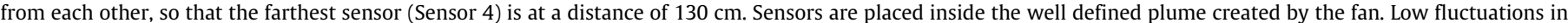
sensor measurements around average values are noticed.

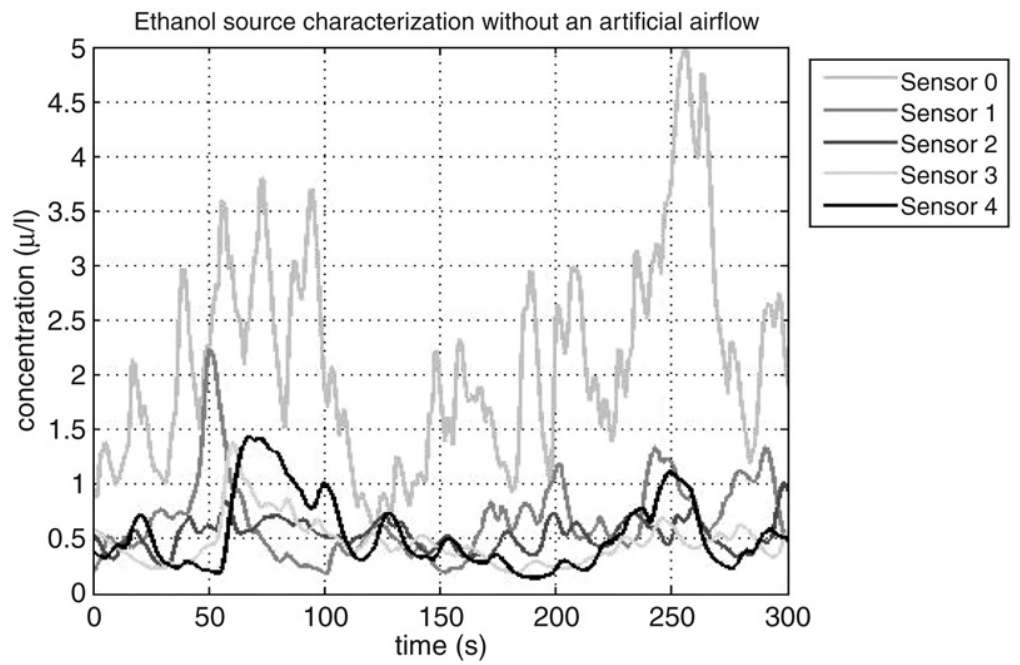

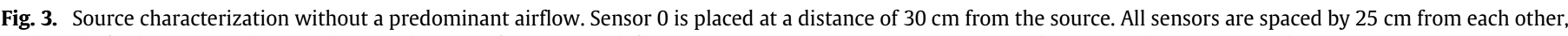
so that the farthest sensor (Sensor 4) is at a distance of $130 \mathrm{~cm}$. High fluctuations in sensor measurements around average values are noticed.

robot performs. Arm lengths have been chosen, based on the search area dimensions. Crosses represent the sampling locations.

At this stage, it is necessary to discuss how to quantify the proximity to a source and to describe the movements of the propagating spiral.

\subsection{Proximity to a source}

Proximity to the source cannot be easily quantified by simply referring to instantaneous gas concentrations, because of the high signal fluctuations. The slow response time of gas sensors makes it even more difficult for the robot to react to instantaneous gas samplings. To solve these problems, SPIRAL uses a stop and sense philosophy to sense the gas. An acquisition is made when the robot is not moving, and lasts $\Delta T$ seconds. After each acquisition, an index, called the Proximity Index (PI), is created.

The index is a number quantifying how close to the source the robot is. Bearing in mind our observations throughout source characterization experiments, we defined the PI, based on the signal average and on the measured concentration peaks, which are a distinctive feature of gas distribution near an emitting chemical source. PI parameters aim at mixing the intensity of the concentration peaks measured and the mean of gas concentration intensity in a weighted sum. The PI is defined as:

$P I=K_{\mu} \cdot \mu+K_{P} \cdot P\left(\Delta T_{P}\right)$

where: $K_{\mu}, K_{p}$ are two multiplicative constant values;

- $\mu$ is the mean of the signal acquired by the gas sensor during the fixed temporal window;

- $P$ is defined as the sum of the number of peaks (a "peak" refers to a local maximum above the average value measured in the acquisition window) of gas concentration, measured in a fixed temporal window $(\Delta T)$, each multiplied by its own intensity. The acquisition temporal window $(\Delta T)$ is divided into intervals of $\Delta T_{p}$ seconds; for each interval, only the highest peak is considered for the final sum;

- $\Delta T_{p}$ is the length of the windows into which the acquisition time is divided.

The mean $(\mu)$ is added to the PI, to catch some information on the distance from the source, when the peaks are low.

The values for these parameters were chosen based on the data coming from our source characterization experiments (in 


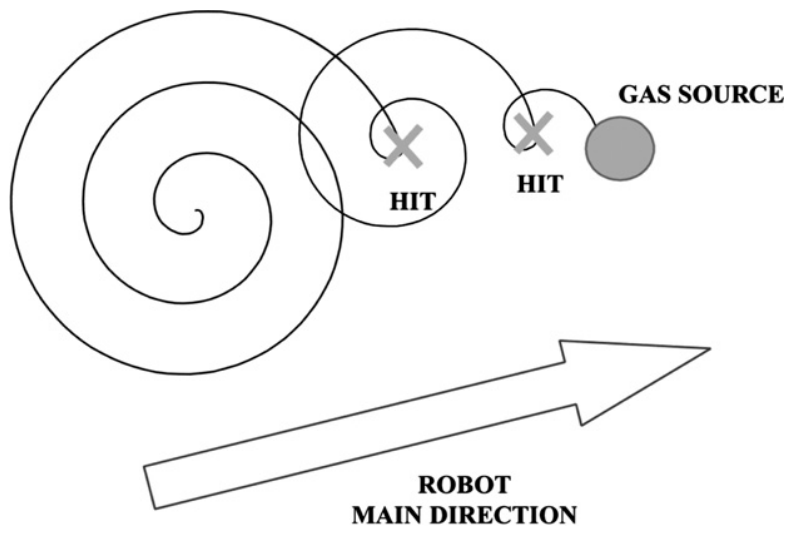

Fig. 4. SPIRAL algorithm basic idea. HIT indicates when the robot figures that it is closer to the source.

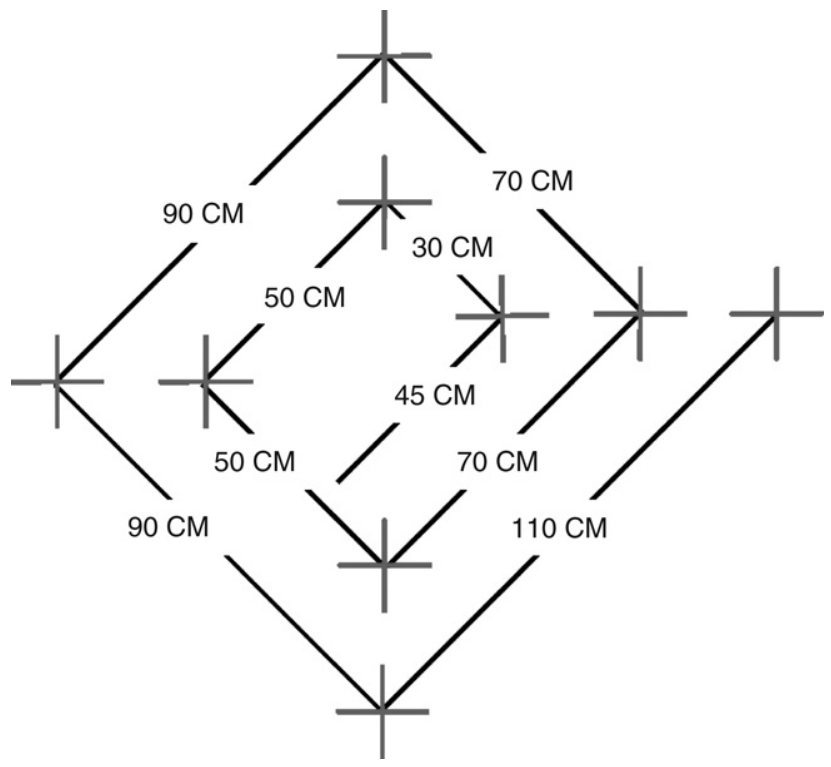

Fig. 5. Actual spiral path covered by the robot. The crosses represent the sampling locations.

the absence of wind). For each sensor, the data acquired were divided into temporal windows of $\Delta T$ seconds. PIs were computed from the different windows. The PI calculation was applied several times, using a different set of parameters each time. The set was generated heuristically within reasonable limit values. The set of parameters that generated better PIs was chosen for the implementation of the algorithm. For "better" we mean a set of parameters that causes the PIs to better follow the order relation between the sensors: that is, given two sensors, the one closer to the source needs to have higher PIs than the one that is further.

The parameters include the following: $\Delta T$ (the fixed acquisition temporal window) is a trade-off between searching algorithm rapidity and PI creation accuracy, and it is fixed at $30 \mathrm{~s}$ (10 s and $20 \mathrm{~s}$ were also tested); $\Delta T_{p}$ is fixed at $5 \mathrm{~s}$ (chosen among the following tested values: $1 \mathrm{~s}, 2 \mathrm{~s}, 5 \mathrm{~s}, 10 \mathrm{~s}, 15 \mathrm{~s}) . \Delta T_{p}$ is a parameter affecting spurious peaks readings. $K_{\mu}$ is fixed at 1 and $K_{p}$ is fixed at $0.5(0.1,0.2,0.5,0.7,1,1.5$ were also tested) (they affect the relative weighting of the average measure and peak intensities). For the rare case in which no peaks are found, $K_{\mu}=2$ is used. This correction is necessary to give more weight to the average value in this specific case. Our experiments suggest that for this value it is reasonable not to have so large a difference between a PI with peaks and a PI without peaks.
The index seems relatively robust to different parameter variations. The most critical parameter to choose is $\Delta T$. It was chosen as a compromise between accuracy in PI creation and speed in finding the source: a high $\Delta T$, in fact, implies PIs that better meet the order relation between the sensors, but it causes an overall increment in the time to find the source.

\subsection{SPIRAL movement strategy}

Drawing inspiration from the behavior of insects, the SPIRAL algorithm reiterates fixed searching in spiral figures. The moth, for example, alternates fixed and repetitive movements to come into contact with odors (casting), and then it moves upwind (surge) [6]. SPIRAL is basically a sort of casting (spiral movements) that is sometimes restarted during the searching task. The decision to restart the spiral movement is made on the basis of the calculated PI values. Obviously, we cannot use a surge movement because we cannot measure a stable and constant wind direction.

The robot moves along a spiral. At the end of each spiral arm, it stops and performs an acquisition. With the data provided by the acquisition, the robot calculates the Proximity Index (PI), which takes into account the intensity of concentration peaks and the mean value of the signal (see Section 3.1 for PI description). The robot has a stored PI value called $T_{P I}$ (Threshold Proximity Index). If the next PI is higher than or equal to the $T_{P I}$, the $T_{P I}$ is refreshed to the new PI value, and a new spiral is started (we call this a HIT). Otherwise, if the new PI is lower than $T_{P I}$, the $T_{P I}$ is not refreshed, and the current spiral is continued (we call this a MISS).

SPIRAL presents the following additional features:

- A minimum threshold for PI: $m T_{P I}$ (Minimum Threshold PI). This threshold is a minimum PI value below which the HIT mechanism is not triggered.

The threshold was chosen based on experiments. We calculated different PIs in different positions in the room used for experiments with the robot, after a source had ejected ethanol for $5 \mathrm{~min}$. Then, PIs calculated at more than $2 \mathrm{~m}$ from the source were considered not to provide sufficient information about the proximity of the source. Their average was selected as the minimum threshold.

- A mechanism of $T_{P I}$ lowering. This mechanism is activated in two different cases:

- five consecutive MISSes; in this case, the $T_{P I}$ parameter is set to the value $m T_{P I}-\Delta$ (where $\Delta$ is a positive constant design parameter);

- three consecutive MISSes with measured PIs lower than $T_{P I} / 2$; also in this case, $T_{P I}$ is set to the value $m T_{P I}-\Delta$.

- The first mechanism aims at solving the problem of a robot moving about in a gas low-concentration area, and the second one aims at preventing spurious high gas measurements (high PIs), which could compromise the SPIRAL efficiency.

An escaping (ESCAPE) movement. When a spiral has ended and no HIT has occurred during the spiral, the robot resets the $T_{P I}$, and then it rotates through a randomly generated angle $\left(45^{\circ}\right.$ granularity). Afterwards, the robot goes forward covering some distance (this distance is a design parameter depending on the geometry of the environment). These movements are intended to explore the environment randomly, when considerable PIs are not produced. Even if this mechanism was rarely triggered in our experiments, it is designed for an application in large indoor environments where areas of low PIs can be found.

An obstacle-collision handling behavior has also been studied and added to SPIRAL. In the case of an obstacle collision, the robot performs a pre-defined sequence of movements, to avoid future obstacle collisions. The obstacle-collision handling behavior is very simple: the robot goes backwards, then rotates, and finally goes 


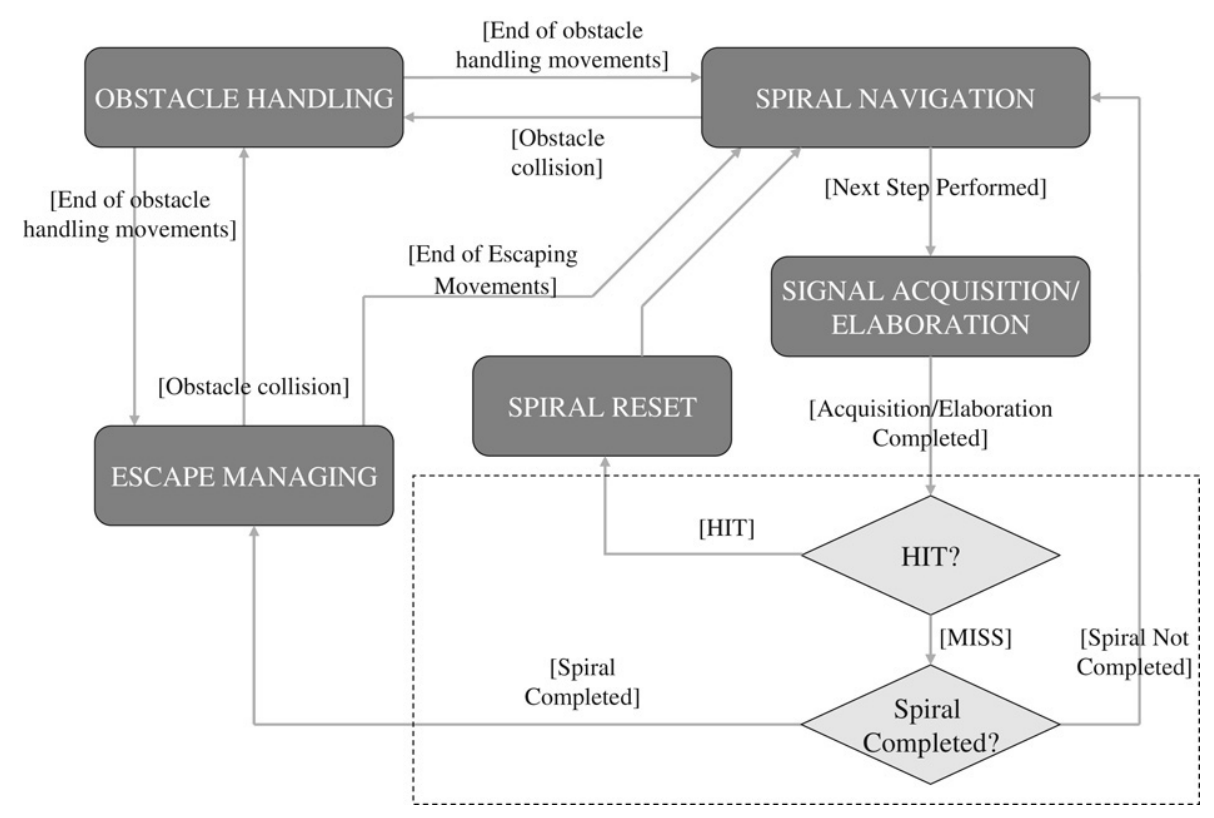

Fig. 6. State diagram of the SPIRAL algorithm.

forward to cover the remaining distance it had to cover before the collision. The sense of rotation (clockwise or counter-clockwise) is chosen by taking into account either the task to continue the spiral movement or the possibility of exploring places with higher PIs. The obstacle collision handling appears to be a fundamental issue above all in an indoor environment with obstacles, or characterized by relatively small dimensions, where possible collisions with the borders of the area may occur.

Fig. 6 reports the state diagram of the SPIRAL algorithm.

\section{Experimental set-up}

The SPIRAL algorithm was implemented on one robot of the MOMO robotic platform, supplied with a TGS 800 , manufactured by Figaro Engineering Inc., a sensor for alcohol detection.

The MOMO platform was developed and presented in previous works of the authors $[9,10]$ to easily test gas-finding algorithms. In our experiments we use one single-agent robot (see Fig. 7), based on the RoboDesigner Kit, manufactured by Japan Robotech Ltd, and a PC. The PC tracks the robot position (via a web-cam) and communicates with the robot, checking its state (through an FM communication channel - an AUR EL XTR-434H from AUREL spa Inc. transceiver is used either with the PC or the robot), and collecting the data produced by the robot's actions. The robot, $17 \mathrm{~cm}$ long and $17 \mathrm{~cm}$ wide, is provided with two encoders on its wheels for rough odometry, a main micro controller that manages movement (PIC 184F52 from Microchip Technology Inc.), two sets of high capacity rechargeable Ni-MH batteries (assuring at least two hours of autonomy), two bumper whiskers as touch sensors, and a TGS 800 gas sensor.

Fig. 8 shows a drawing of the room where the experiments with the robot and preliminary tests with sensors were carried out. The room contained a $3 \mathrm{~m} \times 2.1 \mathrm{~m}$ arena where the robot was able to move. The arena was delimited by polystyrene walls on the north and south sides. Humidity and temperature conditions were assessed so as not to vary remarkably when compared with those reported in the sensor datasheet, and not to affect measurements significantly. During the experiments, the doors and windows of the room were kept shut. In the PC location, two persons were free to move. Fig. 9 shows a picture of the experiment area with the robot searching for the source. During the experiments, the robot

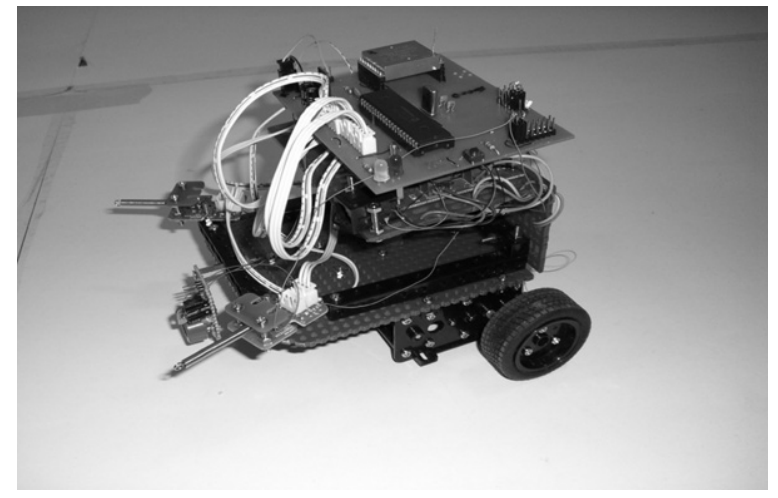

Fig. 7. The MOMO platform overview with one robot.

moved with a speed of about $20 \mathrm{~cm} / \mathrm{s}$. During each acquisition, the robot sampled gas concentration every $1 \mathrm{~ms}$, and it stored averages calculated on $500 \mathrm{~ms}$. A third degree polynomial was used to convert sensor voltage outputs into concentrations during the experiments, and its coefficients were calculated during the sensor calibration experiments (see Section 2).

\section{Experimental results}

This section reports and discusses the experimental results obtained from SPIRAL implementation on a MOMO platform robot.

To evaluate the viability of the proposed SPIRAL algorithm, sets of trials were carried out also implementing other algorithms for the robot movement. Different sets of trials were carried out for each algorithm, for two different starting conditions: $150 \mathrm{~cm}$ and $180 \mathrm{~cm}$ robot-source start distance. A trial was considered completed (stop condition) when the robot reached a $20 \mathrm{~cm} \times$ $20 \mathrm{~cm}$ square containing the source. The trials were divided into sessions: four trials were carried out for each session. Each trial session started five minutes after the opening of the gas source. At the end of each trial session, windows were opened and the air of the room changed. The experiment area is divided into nine sectors (see Fig. 8). The source was placed in sector 6 . The robot starting 


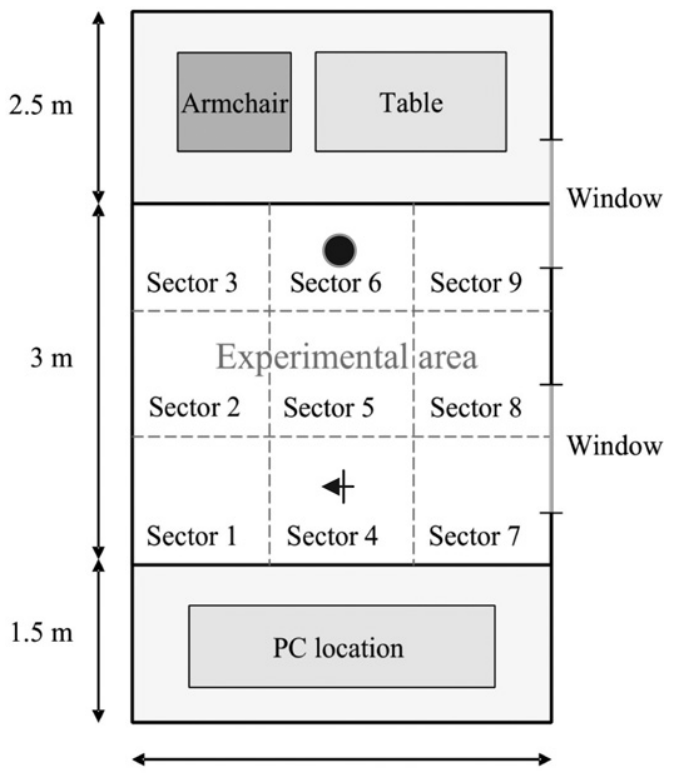

$2.1 \mathrm{~m}$

Fig. 8. A drawing of the experiment room. The position of the source (circle in sector 6) and the starting position of the robot (arrow in sector 4) are showed.

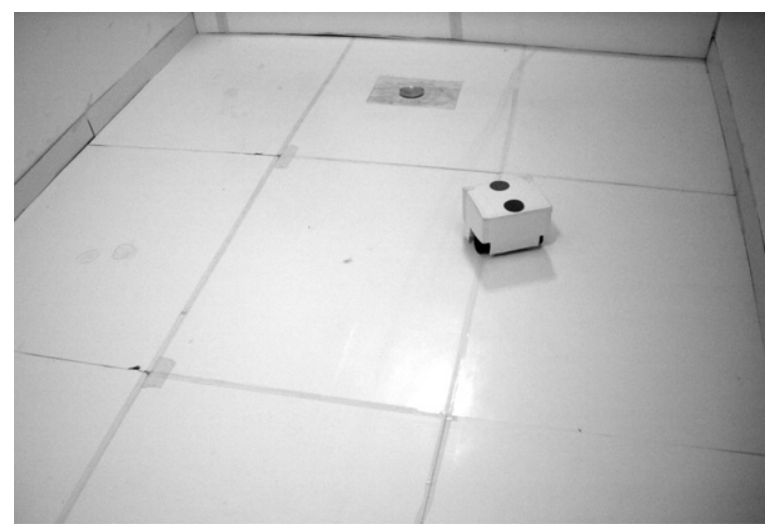

Fig. 9. Experiment area with the robot moving around, with cardboard for webcam tracking.

position was placed in sector 4: the robot is oriented with its nose heading at $90^{\circ}$ to the source. The robot positions were tracked by a webcam, so that searching paths could be easily displayed and post-processed. A typical tracked trial of the SPIRAL algorithm is reported in Fig. 10.

The SPIRAL algorithm functionalities have been compared with two other algorithms: a bacterium-inspired algorithm (BA) [32] and a random SPIRAL algorithm.

The behavior of $E$. coli bacterium [1] consists of straight runs and occasional turns (tumbling). The angles of the turns depend on the sensed chemical spatial gradient computed through successive chemical samplings: if the gradient is positive, the bacterium tends to go straight, otherwise it turns randomly. This behavior has inspired robotic researchers and it has been implemented on robots. We chose this behavior as a comparison with SPIRAL because it is the only algorithm in literature, to the best of the authors' knowledge, which uses only one gas sensor and does not require any information about the wind to work. In this paper, for the first time, we present the results of the bacterium-inspired algorithm application, in an environment with no strong and constant airflow. We adopt the version used in [32]. Description

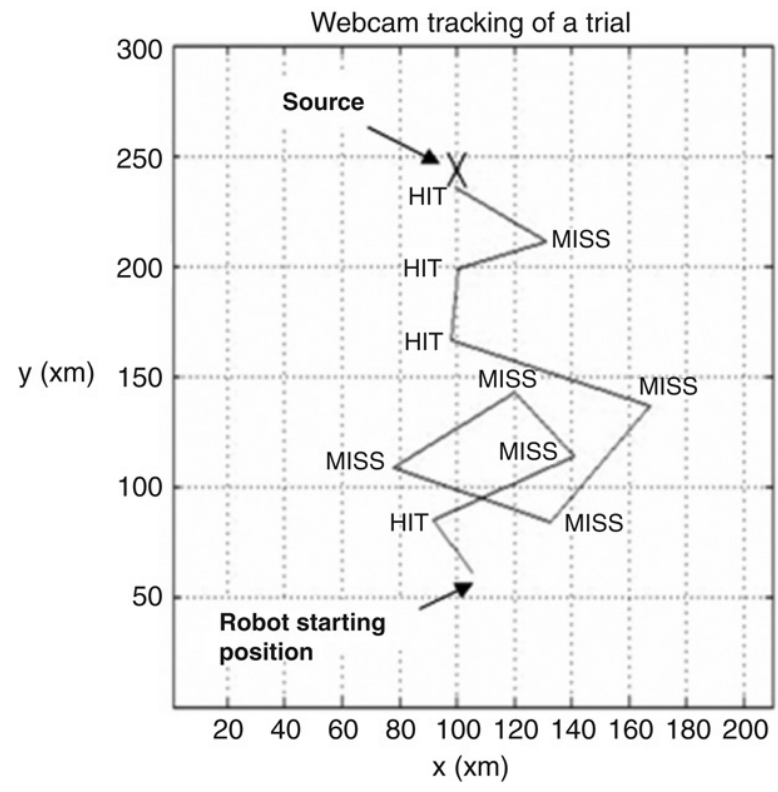

Fig. 10. Webcam tracking of a trial. HIT indicates the condition when the new computed PI is greater than or equal to the stored one. MISS indicates the situation in which the new computed PI is lower than the stored one.

Table 1

Pseudo code of Escherichia coli bacterium algorithm

The E. coli algorithm:

$l=25 \mathrm{~cm}$

repeat \{

if current sensor reading is greater than previous sensor reading

rotate \pm random $\left(5^{\circ}\right)$ and move forward $l \pm \operatorname{random}(0.05 l)$

else

rotate \pm random $\left(180^{\circ}\right)$ and move forward $l \pm$ random $(0.05 l)$

of the implemented E. coli algorithm is given in Table 1. The acquisition time for the BA is set at $3 \mathrm{~s}[26,32]$ which seems to be a suitable time to achieve reliable gas measurements. We also tried to investigate the behavior of BA with an acquisition time of $30 \mathrm{~s}$, the same time as used by SPIRAL.

The random SPIRAL algorithm is implemented in the same way as SPIRAL, but using random values instead of computed Proximity Indices. The SPIRAL random walk was used to assess whether the room dimensions are large enough to avoid a particular kind of random walk can find the source with a number of acquisitions comparable to SPIRAL.

The results (means and standard deviations) of the trials carried out using the three different algorithms are reported in Table 2. The comparison between SPIRAL and random SPIRAL shows clearly the superior efficiency of the proposed method (on average, SPIRAL reaches the source using less than one third of the acquisitions needed by the random SPIRAL algorithm). This assesses that the experimental area is large enough for a "smart" random movement to be unable to achieve comparable results.

SPIRAL works better than the bacterium-inspired algorithm (both with $3 \mathrm{~s}$ and $30 \mathrm{~s}$ acquisition times). The results of our trials with the BA (30 s acquisition time) suggest that, even if the bacterium-inspired algorithm ( $30 \mathrm{~s}$ acquisition time) finds the source with fewer acquisitions than BA ( $3 \mathrm{~s}$ acquisition time), it is much slower. This is due to the nature itself of bacterium-inspired algorithm. The robot needs to perform a remarkable number of acquisitions, in order to orientate itself using the random nature of the bacterium algorithm. Issues come up when the robot moves in areas with low gas concentrations: the high number of acquisitions 
Table 2

Results of the trials with different algorithms

\begin{tabular}{|c|c|c|c|c|}
\hline Algorithm & Starting distance from the source $(\mathrm{cm})$ & Number of trials & Time to find the source (s) & Steps(acquisitions) to find the source \\
\hline \multirow[t]{2}{*}{ SPIRAL } & $\mu=180$ & 15 & $\begin{array}{l}\mu=398.2 \\
\sigma=151.5\end{array}$ & $\begin{array}{l}\mu=11.5 \\
\sigma=4.3\end{array}$ \\
\hline & $\mu=150$ & 15 & $\begin{array}{l}\mu=306.3 \\
\sigma=91.2\end{array}$ & $\begin{array}{l}\mu=9.2 \\
\sigma=2.7\end{array}$ \\
\hline \multirow[t]{2}{*}{ E. COLI (acquisition time = $3 \mathrm{~s}$ ) } & $\mu=180$ & 15 & $\begin{array}{l}\mu=463.3 \\
\sigma=93\end{array}$ & $\begin{array}{l}\mu=67.8 \\
\sigma=14.7\end{array}$ \\
\hline & $\mu=150$ & 15 & $\begin{array}{l}\mu=353.1 \\
\sigma=73.2\end{array}$ & $\begin{array}{l}\mu=50.3 \\
\sigma=11.3\end{array}$ \\
\hline \multirow[t]{2}{*}{ E.COLI (acquisition time $=30 \mathrm{~s}$ ) } & $\mu=180$ & 4 & $\begin{array}{l}\mu=1671.2 \\
\sigma=134.9\end{array}$ & $\begin{array}{l}\mu=49.3 \\
\sigma=4.1\end{array}$ \\
\hline & $\mu=150$ & 4 & $\begin{array}{l}\mu=1184.1 \\
\sigma=158.1\end{array}$ & $\begin{array}{l}\mu=35.7 \\
\sigma=5.1\end{array}$ \\
\hline \multirow[t]{2}{*}{ SPIRAL RANDOM } & $\mu=180$ & 10 & $\begin{array}{l}\mu=1351 \\
\sigma=291\end{array}$ & $\begin{array}{l}\mu=38.6 \\
\sigma=8.1\end{array}$ \\
\hline & $\mu=150$ & 10 & $\begin{array}{l}\mu=991 \\
\sigma=193\end{array}$ & $\begin{array}{l}\mu=28.3 \\
\sigma=5.2\end{array}$ \\
\hline
\end{tabular}

needed to approach areas closer to the source where the algorithm can work better make the task of moving towards areas with higher concentrations too time consuming. Time to find the source drastically decreases reducing the sampling time. The BA $(3 \mathrm{~s}$ acquisition time version) produces times to find the source that are comparable with SPIRAL times. However, SPIRAL works better. It is worth noting that the obstacle handling behavior becomes above all fundamental in BA experiments. In fact, BA (3 s acquisition time version) is more easily affected than SPIRAL by turbulent effects, and it tends to wander about hitting the borders of the experimental area. The many collisions with the borders and the consequent collision handling behavior help the robot to explore the experimental area and makes it able to reach areas with higher gas concentrations (closer to the source), where the BA algorithm is more effective. In the trials in which SPIRAL was used, a few collisions against the borders were observed (see for example Fig. 10). This suggests that enlarging the experimental area would not change SPIRAL results significantly, whereas it would decrease BA efficiency: the robot might move in low gas concentration areas and it could come closer to the source only after a long time. SPIRAL balances its greater robustness against turbulent effects with a longer acquisition window.

\section{Conclusions and future works}

This paper focuses on the design and implementation of a biologically-inspired algorithm on a robotic platform, with the purpose of localizing a gas source in an indoor environment in the absence of strong airflows. Turbulence and weak convective flows strongly characterize the dispersion process. Gas is present in "packets", and high fluctuations around a mean value are observed (see Fig. 3). These problems decrease with the introduction of a strong wind. A strong and constant wind increases the energy of turbulent mixing producing an area relatively close to the source where a gradient is detectable and low fluctuations around a mean value are present (see Fig. 2). Without a strong wind, information about airflow direction cannot be used to infer the position of the ejecting source. Therefore, searching an odor source in a windless room becomes a harder task. In this paper, we focused on this environment for two different reasons: it can well represent a realistic indoor scenario, and these conditions may be peculiar to areas behind obstacles also when a strong mean flow is present in the searching area.

To perform gas source localization in these experiment conditions, we proposed the SPIRAL algorithm, which consists of spiral movements and stop and acquisition steps, after which a Proximity Index is created. The Proximity Index aims at expressing proximity to the source with a numerical value. The index includes the measured gas peak intensity and the signal average as well. SPIRAL allows the robot to move towards higher and higher Proximity Index locations, which correspond, on average, to locations closer to the source.

The SPIRAL algorithm was implemented on the MOMO robotic platform $[9,10]$ and tested by means of experiments with one robot. The validity of the SPIRAL approach was assessed by comparing it with an E. coli bacterium algorithm (the only other method available in the literature, to the best of the authors' knowledge, which presents the same features as SPIRAL: single and not differential gas measurements and no use of information about the wind) and a SPIRAL random-walk. The results are very encouraging and show that the algorithm is robust and reliable in finding the source. The SPIRAL algorithm main features can be summarized as follows:

- the robot moves spirally towards high Proximity Index locations;

- the spiral movements make the algorithm intrinsically robust, which means that wrong measurements (low measurements near the source) do not compromise its effectiveness. If no trigger occurs, the spiral movement, in fact, allows the agent to pass again in a location closer to the source, where the robot has another possibility to trigger;

- no differential measurements are needed. There is no necessity to calibrate more than one sensor: to calibrate different gas sensors is not an easy task. More importantly, differential measurements with spatially separated sensors might be misleading, because of gas concentration high fluctuations;

- it works without a constant forced airflow and without the necessity of acquiring information about the wind (measuring wind direction in the environment described may be hard to accomplish [19]).

In the literature, only a few works address the gas source localization issue in the environment analysed in this paper. The only other method showing the same features, to the best of the authors' knowledge, is the bacterium-inspired algorithm. From our experiments SPIRAL appears to be more robust than the bacteriuminspired method.

If we take the time used to find a source as a merit factor, SPIRAL needs more time if compared with the time necessary for the algorithms available in the literature, which use the wind information. However, the time used is comparable and of the same order of magnitude. Similar results achieved in a more difficult experiment setting show the viability of SPIRAL as an algorithm to be used in an indoor environment, when the airflow is so weak or unsettled that a state-of-the-art anemometer 
cannot measure it reliably. It could also be used together with traditional algorithms in areas (above all behind obstacles) where traditional algorithms fail because of the difficulty of measuring wind direction.

In future works, further experiments in larger areas will be carried out, to investigate the efficiency of SPIRAL. In addition, we will use SPIRAL in a multi-source and windless environment, together with a probabilistic mapping algorithm [11]. Once the presence of a source in an area has been most likely detected, the spiral movements of SPIRAL may be used to localize the ejecting source.

\section{References}

[1] J. Adler, The sensing of chemicals by bacteria, Scientific American 234 (4) (1976) $40-47$.

[2] A. Arbas, M.A. Willis, R. Kanzaki, Organization of goal-oriented locomotion: Pheromone-modulated flight behavior of moths, in: R.D. Beer, R.E. Ritzmann, T. McKenna (Eds.), Biological Neural Networks in Invertebrate Neuroethology and Robotics, Academic Press, San Diego, 1993, pp. 159-198.

[3] J. Atema, Eddy chemotaxis and odor landscapes exploration of nature with animals sensors, Biological Bulletin 191 (1996) 129-138.

[4] E. Balkovsky, B.I. Shraiman, Olfactory search at high Reynolds number, Proceedings of the National Academy of Science 99 (20) (2002) 12589-12593.

[5] J. Basil, J. Atema, Lobster orientation in turbulent odor plumes: Simultaneous measurements of tracking behavior and temporal odor patterns, Biological Bulletin 187 (1994) 272-273.

[6] J.H. Belanger, M.A. Willis, Biologically-inspired search algorithms for locating unseen odor sources, in: Proceedings of the IEEE ISIC/CIRA/ISAS Joint Conference Galthersburg, MD, USA, 1998, pp. 265-270.

[7] D.B. Dusenbery, Sensory Ecology: How Organisms Acquire and Respond to Information, Freeman, New York, 1992.

[8] L. Edelstein-Keshet, J. Watmough, G.B. Ermentrout, Trail following in ants: Individual properties determine population behaviour, Behavioral Ecology and Sociobiology 36 (2) (1995) 119-133.

[9] G. Ferri, E. Caselli, V. Mattoli, A Mondini, B. Mazzolai, P. Dario, A biologicallyinspired algorithm implemented on a new highly flexible multi-agent platform for gas source localization, in: Proceedings of the IEEE RAS-EMBS International Conference on Biomedical Robotics and Biomechatronics Biorob 2006, Pisa, Italy, 2006, pp. 573-578.

[10] G. Ferri, E. Caselli, V. Mattoli, A. Mondini, B. Mazzolai, P. Dario, A biologicallyinspired algorithm for gas/odor source localization in an indoor environment with no strong airflow: First experimental results, in: Proceedings of Workshop on Robotic Olfaction of IEEE International Conference on Robotics and Automation, ICRA 2007, Rome, Italy, 2007.

[11] G. Ferri, M.V. Jakuba, E. Caselli, V. Mattoli, B. Mazzolai, D.R. Yoerger, P. Dario, Localizing multiple gas/odor sources in an indoor environment using Bayesian occupancy grid mapping, in: Proceedings of the IEEE/RSJ International Conference on Intelligent Robots and Systems, IROS 2007, San Diego, CA, USA 2007.

[12] F.W. Grasso, T.R. Consi, D.C. Mountain, J. Atema, Biomimetic robot lobster performs chemo-orientation in turbulence using a pair of spatially separated sensors: Progress and challenges, Robotics and Autonomous Systems 30 (2000) 115-131.

[13] F.W. Grasso, J.A. Basil, How lobsters, crayfishes, and crabs locate sources of odor current perspectives and future directions, Current Opinion in Neurobiology 12 (2002) 721-727.

[14] L. Greenberg, Genetic component of bee odor in kin recognition, Science 30 206 (4422) (1979) 1095-1097.

[15] E. Hancock, A primer on smell, John Hopkins Magazine 9 (September) (1996) (Electronic Edition - A Special Issue on the Senses).

[16] A.T. Hayes, A. Martinoli, R.M. Goodman, Distributed odor source localization, IEEE Sensors Journal 2 (3) (2002) 260-271.

[17] H. Ishida, G. Nakayama, T. Nakamoto, T. Moriizumi, Controlling a gas/odor plume-tracking robot on transient responses of gas sensors, IEEE Sensors Journal 5 (3) (2005) 537-545.

[18] H. Ishida, H. Tanaka, H. Taniguchi, T. Moriizumi, Mobile robot navigation using vision and olfaction to search for a gas/odor source, Autonomous Robots 20 (2006) 231-238.

[19] H. Ishida, Robotic systems for gas/odor source localization: Gap between experiments and real-life situations, in: Proceedings of Workshop on Robotic Olfaction of IEEE International Conference on Robotics and Automation, ICRA 2007, Rome, Italy, 2007.

[20] S. Kazadi, R. Goodman, D. Tsikata, D. Green, H. Lin, An autonomous water vapor plume tracking robot using passive resistive polymer sensors, Autonomous Robots 9 (2000) 175-188.
[21] A. Lilienthal, M.Zell, U. Wandel, Sensing odour sources in indoor environments without a constant airflow by a mobile robot in: Proceedings of the 2001 IEEE International Conference on Robotics and Automation, ICRA 2001, Seoul, Korea, 2001, pp. 4005-4010.

[22] A. Lilienthal, T. Duckett, Experimental analysis of smelling Braitenberg vehicles, in: Proceedings of the IEEE International Conference on Advanced Robotics, ICAR 2003, Coimbra, Portugal, 2003, pp. 375-380.

[23] A. Lilienthal, D. Reiman, A. Zell, Gas source tracing with a mobile robot using an adapted moth strategy, Autonome Mobile Systeme (AMS) Karlsruhe, December 4-5, (2003) 150-160.

[24] A. Lilienthal, T. Ducket, Building gas concentration gridmaps with a mobile robot, Robotics and Autonomous Systems 48 (2004) 3-16.

[25] A. Lilienthal, A. Loutfi, T. Duckett, Airborne chemical sensing with mobile robots, Sensors 6 (2006) 1616-1678.

[26] C. Lytridis, E.E. Kadar, G.S. Virk, A systematic approach to the problem of odour source localization, Autonomous Robots 20 (2006) 261-276.

[27] L. Marques, U. Nunes, A. de Almeida, Olfaction-based mobile robot navigation, Thin Solid Films 418 (2002) 51-58.

[28] J. Murlis, J.S. Elkinton, R.T. Cardè, Odor plumes and how insects use them, Annual Review of Entomology 37 (1992) 505-532.

[29] P. Pyk, S. Bermudez i Badia, U. Bernardet, P. Knusel, M. Carlsson, J. Gu, E. Chanie, B.S. Hansson, T.C. Pearce, P.F.M.J. Verschure, An artificial moth: Chemical source localization using a robot based neuronal model of moth optomotor anemotactic search, Autonomous Robots 20 (2006) 197-213.

[30] R.A. Russell, Laying and sensing odor markings as a strategy for assisting mobile robot navigation trasks, IEEE Robotics and Automation Magazine 2 (1995) 3-9.

[31] R.A. Russell, D. Thiel, R. Deveza, A. Mackay-Sim, A robotic system to locate hazardous chemical leaks, in: Proceedings of International Conference on Robotics and Automation, ICRA 1995, 1, Nagoya, Japan, 1995, pp. 556-561.

[32] R.A. Russell, A. Bab-Hadiashar, R.L. Scheffer, G. Wallace, A comparison of reactive chemotaxis algorithms, Robotics and Autonomous Systems 45 (2003) 83-97.

[33] B.I. Shraiman, B.I. Siggia, Scalar turbulence, Nature 405 (6787)(2000) 639-646.

[34] N.J. Vickers, Mechanisms of animal navigation in odor plumes, Biological Bulletin 198 (2) (2000) 203-212.

[35] M.R. Wandel, A. Lilienthal, T. Duckett, U. Weimar, A. Zell, Gas distribution in unventilated indoor environments inspected by a mobile robot, in: Proceedings of the IEEE International Conference on Advanced Robotics, ICAR 2003, Coimbra, Portugal, 2003, pp. 507-512.

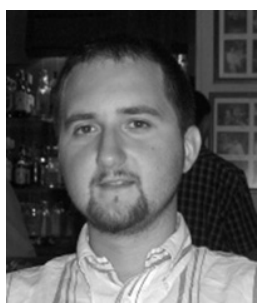

Gabriele Ferri received the Laurea degree (M.S.) in Computer Engineering (with Honors) from the University of Pisa, Italy, in 2003. From 2003 to 2005, he worked as a Software/System Consultant Engineer for WASS company (a Finmeccanica group company) in Livorno, Italy, working on the design of a new System of Control and Guidance for a light-weighted torpedo. He is currently a Ph.D. candidate in Biorobotic Science and Engineering at IMT Advanced Studies in Lucca, Italy, an inter-university consortium formed also by Scuola Superiore S.Anna. From November 2006 he has been a visiting researcher at Woods Hole Oceanographic Institution, Woods Hole, Massachusetts, USA. His research interests include robotic systems for environmental monitoring/exploration, control theory and robot navigation issues.

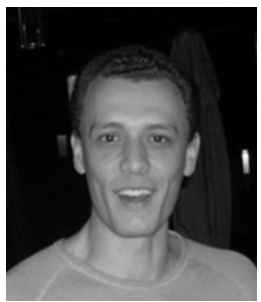

Emanuele Caselli received his Laurea degree (M.S.) in Electronic Engineering from the University of Modena, Italy in 2004. He is currently a Ph.D. candidate in Biorobotic Science and Engineering at IMT Advanced Studies Institute in Lucca, Italy. From 2005 he worked in collaboration with Scuola Superiore Sant'Anna, Italy. His research interests include the implementation of robotic swarms systems for environmental monitoring and exploration, sensors integration, intra-robot communication and developing of swarm behaviour.

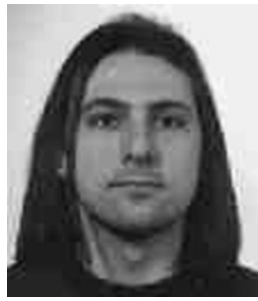

Virgilio Mattoli received his Laurea degree in chemistry (with Honors) from the University of Pisa and the Diploma in Chemistry (with Honors) from the Scuola Normale Superiore of Pisa in 2000. He joined the CRIM Lab of Scuola Superiore Sant'Anna in 2001 with a research program focused on the control and integration of miniaturized devices for environmental and biomedical application. He received his Ph.D. in bio-engineering (with Honors) in 2005. He has been a visiting researcher at Stanford University, California, USA, and at Waseda University, Tokyo, Japan. His main research interests include: thin film sensor microfabrication, sensor conditioning, miniaturized acquisition system, wireless sensor networks, mechatronics and biorobotics. He is currently involved in several research projects on these topics. 


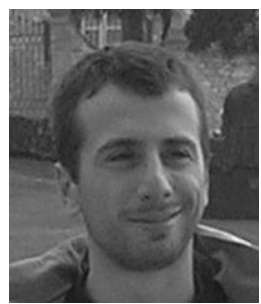

Alessio Mondini received is Laurea degree in electronic engineering from the University of Pisa, Italy in 2002. In 2003, he worked in the national energy company (ENEL) with a stage program focused on data acquisition system and sensor conditioning for physical measures into the geothermal wells. From May 2004, he is working at CRIM Laboratory of Scuola Superiore Sant'Anna. His main research interests include microsystems technologies, microelectronics and miniaturized data acquisition system for sensor networks.

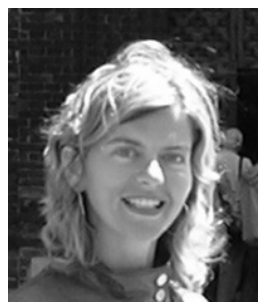

Barbara Mazzolai received her Laurea degree in Biology (with Honors) from the University of Pisa, Italy, in 1995 From 1994 to 1998, she worked at the Institute of Biophysics of the National Research Council on environmental topics and in particular, on mercury pollution. In 1998, she received her Master's degree in Eco-Management and Audit Schemes (EMAS) from Scuola Superiore Sant'Anna (SSSA), Pisa, Italy. Then, she had a research assistant position at Center for Research in Microengineering (CRIM) Laboratory of SSSA from February 1999 until June 2004. From July 2004, she obtained a temporary position of assistant professor in bioengineering at SSSA. Her current research interests include robotics, autonomous robots, microsystems and sensors for environmental, agrofood and biomedical applications. She is currently working on several European and international projects focused on these fields.

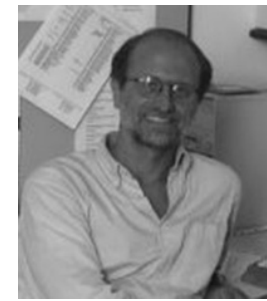

Paolo Dario received the Dr. Eng. degree in mechanical engineering from the University of Pisa, Pisa, Italy, in 1977. He is currently a Professor of Biomedical Robotics at the Scuola Superiore Sant'Anna, Pisa, Italy. He also teaches courses at the School of Engineering of the University of Pisa, and at the Campus Biomedico University, Rome, Italy. He has been a Visiting Professor at Brown University, Providence, RI, at the Ecole Polytechnique Federale de Lausanne (EPFL), Lausanne, Switzerland, and at Waseda University, Tokyo, Japan. He was the founder of the Advanced Robotics Technologies and Systems (ARTS) Laboratory and is currently the co-cordinator of the Center for Research in Microengineering (CRIM) Laboratory of the Scuola Superiore Sant'Anna, where he supervises a team of about 70 researchers and Ph.D. students. He is also the Director of the Polo Sant'Anna Valdera and a Vice-Director of the Scuola Superiore Sant'Anna. His main research interests are in the fields of medical robotics, mechatronics, and micro/nanoengineering, and specifically in sensors and actuators for the above applications. He is the coordinator of many national and European projects, the editor of two books on the subject of robotics, and the author of more than 200 scientific papers (75 in ISI journals). He is Editor-in-Chief, Associate Editor, and Member of the Editorial Board of many international journals. Prof. Dario served as President of the IEEE Robotics and Automation Society during 2002-2003, and he is currently Co-Chair of the Technical Committees on Bio-robotics and of Roboethics of the same society. He is a Fellow of the European Society on Medical and Biological Engineering, and a recipient of many honors and awards, such as the Joseph Engelberger Award. He is also a Member of the Board of the International Foundation of Robotics Research (IFRR). 